Iris Campos Martins

\title{
Projeto de Serviços: proposta de modelo teórico para sites de compras coletivas
}

Dissertação apresentada como requisito parcial para obtenção do grau de Mestre pelo Programa de Pós-Graduação em Engenharia de Produção do Departamento de Engenharia Industrial da PUC-Rio.

Orientadora: Profa. Fernanda Maria Pereira Raupp Coorientadora: Profa. Suzana Quinet de Andrade Bastos 
Iris Campos Martins

\title{
Projeto de Serviços: proposta de modelo teórico para sites de compras coletivas
}

Dissertação apresentada como requisito parcial para obtenção do grau de Mestre pelo Programa de Pós-Graduação em Engenharia de Produção da PUC-Rio. Aprovada pela Comissão Examinadora abaixo assinada.

\author{
Profa. Fernanda Maria Pereira Raupp \\ Orientadora \\ Departamento de Engenharia Industrial - PUC-Rio
}

Prof?. Suzana Quinet de Andrade Bastos

Co-Orientadora

Universidade Federal de Juiz de Fora - UFJF

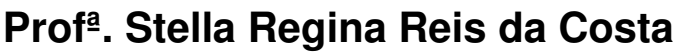

Universidade Federal Rural do Rio de Janeiro - UFRRJ

Prof $f^{\mathrm{a}}$. Adriane Monteiro Cavalieri Barbosa Instituto Nacional de Tecnologia - INT

Prof. José Eugenio Leal

Coordenador Setorial do Centro Técnico Científico - PUC-Rio

Rio de Janeiro, 16 de abril de 2012 
Todos os direitos reservados. É proibida a reprodução total ou parcial do trabalho sem autorização da universidade, da autora e do orientador.

\section{Iris Campos Martins}

Possui graduação em Ciências Econômicas pela Universidade Federal de Juiz de Fora (2008) e Pós Graduação em Métodos Estatísticos pela mesma Instituição (2009). Atuação na área de Economia, com experiência em docência em Introdução à Teoria Econômica e monitoria de Economia Monetária, projeto de pesquisa/iniciação científica em trabalhos de análise espacial e logística territorial. Participação no VIII Intercâmbio Acadêmico da Secretaria de Acompanhamento Econômico do Ministério da Fazenda.

Ficha Catalográfica

Martins, Iris Campos

Projeto de serviços: proposta de modelo teórico para sites de compras coletivas / Iris Campos Martins; orientadora: Fernanda Maria Pereira Raupp; co-orientadora: Suzana Quinet de Andrade Bastos. - 2012.

$112 \mathrm{f} . ; 30 \mathrm{~cm}$

Dissertação (mestrado)-Pontifícia Universidade Católica do Rio de Janeiro, Departamento de Engenharia Industrial, 2012.

Inclui bibliografia

1. Engenharia Industrial - Teses. 2. Projeto e desenvolvimento de serviços. 3. Modelos de referência. 4. Sites de compras coletivas. I. Raupp, Fernanda Maria Pereira. II. Bastos, Suzana Quinet de Andrade. III. Pontifícia Universidade Católica do Rio de Janeiro. Departamento de Engenharia Industrial. IV. Título.

CDD: 658.5 
Minha mãe, meu exemplo de dedicação ao estudo e à pesquisa. 


\section{Agradecimentos}

À Profa. Dra. Fernanda Maria Pereira Raupp, por exercer a orientação com dedicação em cada uma das fases do estudo, contribuindo para o aprendizado contínuo e consolidação do trabalho, e pela confiança ao ter me deixado trilhar os caminhos do conhecimento de modo respeitoso e paciente.

À Profa. Dra. Suzana Quinet de Andrade Bastos, coorientadora, que com competência e pertinentes observações, proporcionou oportunidades de melhoria para o trabalho. E pelo convívio novamente através de mais uma orientação, tornando capaz a conquista de mais um degrau da minha carreira acadêmica.

Às Professoras Dra. Adriane Monteiro Cavalieri Barbosa e Dra. Stella Regina Reis da Costa por gentilmente terem aceitado o convite de participarem da minha banca e contribuírem com suas experiências / conhecimentos para o enriquecimento desse trabalho.

Aos Professores do Departamento de Engenharia Industrial da Pontifícia Universidade Católica do Rio de Janeiro, por todo o conhecimento passado e pela oportunidade de novos aprendizados.

Ao CNPq pelo apoio financeiro, auxilio necessário para que este estudo fosse possível.

À minha mãe para quem também dedico este trabalho, por ser minha fonte inesgotável de exemplos a seguir na minha construção pessoal e profissional.

Ao meu noivo por ter caminhado ao meu lado nestes dois anos de estudo, compreendendo o meu momento e por ter me apoiado sempre.

Aos meus irmãos que mesmo na distância sempre se fazem presentes. 
A todos os colegas do programa, pela troca de experiências. E aos meus amigos queridos que de maneira indireta e não menos importante me ajudaram a concluir este projeto.

Por fim, a todos que de alguma maneira contribuíram para que receber este título fosse possível. 


\section{Resumo}

Martins, Iris Campos; Raupp, Fernanda Maria Pereira. Projeto de Serviços: proposta de modelo teórico para sites de compras coletivas. Rio de Janeiro, 2012. 112p. Dissertação de Mestrado - Departamento de Engenharia Industrial, Pontifícia Universidade Católica do Rio de Janeiro.

Esta dissertação tem como objetivo principal responder ao seguinte questionamento: existe um modelo teórico para o Projeto de Serviços que possa ser aplicado por uma empresa prestadora de serviços de compras coletivas? Para esta análise foi feita uma ampla revisão sobre os modelos existentes, bem como sobre a unidade de investigação, o objeto desse estudo, os Sites de Compras Coletivas. Partindo de uma pesquisa descritiva, este estudo propõe em última análise um modelo esquemático para o projeto de serviço de um site de compras coletivas, o qual representa o mundo atual globalizado e interligado através dos meios de comunicação, via internet. Como resultado do trabalho cabe destacar que a elaboração do projeto para os Sites de Compras Coletivas permitiu que este englobasse três fatores que aumentam as suas chances de sucesso. Primeiro, o serviço se torna orientado para o mercado, no caso para os consumidores virtuais. Segundo, a realização de minuciosos estudos de viabilidade e especificação que consideram as características do público alvo, o mercado consumidor, de cada oferta e da concorrência. E, por fim, o projeto dá maior atenção para a qualidade do desenvolvimento do serviço, através das informações que permitam corrigir ou melhorar seu desempenho.

\section{Palavras-chave}

Projeto e desenvolvimento de serviços; Modelos de referência; Sites de compras coletivas. 


\section{Abstract}

Martins, Iris Campos; Raupp, Fernanda Maria Pereira (Advisor). Design Services: proposal of theoretical model for collective buying sites. Rio de Janeiro, 2012. 112p. Dissertation - Departamento de Engenharia Industrial, Pontifícia Universidade Católica do Rio de Janeiro.

This dissertation has as main purpose to answer the following question: is there an existing theoretical model for Project Services that can be applied by a service provider of collective purchasing? For this analysis a comprehensive review of the existing models was conducted, as well as of the research unit, the object of this study, the Collective Purchasing Sites. Starting from a descriptive research, this study ultimately proposes a schematic model for the project service of a collective purchasing site, which represents the current globalized and interconnected world through the media, via internet. As a result of the work, it is worth noting that the project design for Collective Purchasing Sites enables to encompass three factors that increase its chances of success. First, the service becomes oriented to the market, in this case to the virtual consumers. Second, it conducts feasibility studies and detailed specification that consider the characteristics of the target audience, the consumer market, of each offer and the competition. And, at last, the project gives much attention to the quality of service development through the information to correct or improve its performance.

\section{Keywords}

Project and development of services; Reference models; Collective purchasing sites. 


\section{Sumário}

1 Introdução 13

1.1 Contextualização do Problema 13

$\begin{array}{ll}1.2 \text { Objetivo } & 15\end{array}$

1.2.1 Objetivo Principal 15

$\begin{array}{ll}1.2 .2 \text { Objetivos Específicos } & 16\end{array}$

$\begin{array}{ll}1.3 \text { Relevância } & 16\end{array}$

$\begin{array}{ll}1.4 \text { Delimitações do estudo } & 17\end{array}$

$\begin{array}{lr}\text { 1.4.1 Quanto ao escopo } & 17\end{array}$

$\begin{array}{ll}\text { 1.4.2 Quanto à localidade } & 17\end{array}$

$\begin{array}{ll}\text { 1.4.3 Quanto ao tempo } & 17\end{array}$

$\begin{array}{ll}1.5 \text { Estrutura do trabalho } & 18\end{array}$

2 Referencial teórico 19

2.1 Concepção do projeto de serviços $\quad 19$

2.1.1 Definição de serviço 19

$\begin{array}{ll}2.1 .2 & \text { O projeto de serviço } \\ \end{array}$

2.1.3 Etapas do projeto da concepção de serviços 40

2.1.3.1 Análise estratégica $\quad 41$

2.1.3.2 Geração e seleção de ideias para o serviço 48

2.1.3.3 Definição do pacote de serviços 50

2.1.3.4 Definição das especificações do serviço 51

2.1.4 Projeto dos processos do serviço 51

2.1.4.1 Mapeamento e controle dos processos de serviço 53

2.1.5 Projeto das instalações do serviço 55

2.1.6 Avaliação e melhoria do serviço 56

2.1.7 Marketing no Projeto de Serviços $\quad 57$

2.1.8 Inovação em serviços $\quad 59$

2.2 Modelos de projeto e desenvolvimento de serviços 63 
3 Metodologia da pesquisa

3.1 Tipo de pesquisa

3.2 Caracterização da unidade de investigação: os sites de compras coletivas

3.3 Coleta de dados $\quad 71$

3.4 Análise dos dados $\quad 71$

4 Análise dos modelos para desenvolvimento de projeto de serviços e dos sites de compras coletivas 73

4.1 Pontos fortes e gargalos dos modelos para desenvolvimento de projeto de serviços 73

4.2 Especificação do serviço 81

4.2.1 Escolha dos produtos e ou serviços mais promissores para promoção 86

4.2.2 Contratar o site de compra coletiva 86

4.2.3 Definir os parâmetros da campanha de compra coletiva e preparar para atender a demanda mantendo o padrão

5 Caracterização da unidade de investigação: Os sites de compras coletivas

5.1 Agentes envolvidos e etapas $\quad 89$

5.1.1 Projeto da concepção do serviço 90

5.1.1.1 Avaliação estratégica (análise do mercado e dos objetivos coorporativos)

90

5.1.1.2 Geração e seleção de ideias (a partir de fontes internas e externas) 91

5.1.1.3 Definição das especificações (atributos) 91

5.1.1.4 Definição do pacote e do conceito do serviço 91

5.1.2 Projeto do processo do serviço 92

5.1.3 Projeto das instalações do serviço 93

5.1.4 Avaliação e melhoria do serviço e recuperação de falhas 93

5.1.5 Avaliação da viabilidade 94

5.1.6 Projeto do plano de marketing $\quad 94$ 
6 Modelo proposto: projeto de serviços dos sites de compras coletivas 96

7 Conclusões e considerações finais

8 Referências 


\section{Lista de figuras}

Figura 1. Determinantes da qualidade dos serviços 32

Figura 2. Componentes da qualidade em serviços 33

Figura 3. Modelo para projeto e desenvolvimento de serviços 36

Figura 4. Modelo conceitual do sistema de operações de serviços $\quad 36$

Figura 5. Projeto dos processos de serviço $\quad 37$

Figura 6. Formulação da estratégia de operações 43

Figura 7. Etapas do Planejamento de Steiner 45

Figura 8. Três grandes estratégias básicas 47

Figura 9. Matriz da Análise SWOT e seus resultados 48

Figura 10. Modulariedade da sistemática da inovação de acordo com os objetivos de cada fase $\quad 49$

Figura 11. Três elementos acrescidos ao Mix de Marketing para Serviços $\quad 58$

Figura 12. Triângulo de Albrecht 64

Figura 13. Modelo de desenvolvimento de novos serviços 75

Figura 14. Ciclo de planejamento para integração de novos serviços 77

Figura 15. Modelo para projeto e desenvolvimento de serviços 80

Figura 16. Agentes envolvidos na prestação do serviço de compras coletivas 90

Figura 17. Modelo para Projeto e desenvolvimento de Serviços dos Sites de Compra Coletiva 101 